\title{
ON RINGS FOR WHICH HOMOGENEOUS MAPS ARE LINEAR
}

\author{
P. FUCHS, C. J. MAXSON, AND G. PILZ
}

(Communicated by Maurice Auslander)

\begin{abstract}
Let $\mathscr{R}$ be the collection of all rings $R$ such that for every $R$ module $G$, the centralizer near-ring $M_{R}(G)=\{f: G \rightarrow G \mid f(r x)=r f(x)$, $r \in R, x \in G\}$ is a ring. We show $R \in \mathscr{R}$ if and only if $M_{R}(G)=\operatorname{End}_{R}(G)$ for each $R$-module $G$. Further information about $\mathscr{R}$ is collected and the Artinian rings in $\mathscr{R}$ are completely characterized.
\end{abstract}

\section{INTRODUCTION}

Let $R$ be a ring with identity and $G$ a unitary left $R$-module. The set $M_{R}(G):=\{f: G \rightarrow G \mid f(r x)=r f(x), r \in R, x \in G\}$ is a zero-symmetric nearring with identity under the operations of function addition and composition. If $G=R, M_{R}(R) \cong R$ so $M_{R}(R)$ is a ring. If $R$ is a field and $G=R^{2}$ then it is known that $M_{R}\left(R^{2}\right)$ is not a ring [3]. On the other hand, when $R$ is a finite simple ring, but not a field, it was found in [2] that $M_{R}(G)$ is a ring for each finite $R$-module $G$. In this paper we investigate two questions raised by the above remarks; namely, (1) characterize those rings $R$ such that $M_{R}(G)$ is a ring for every $R$-module $G$ and (2) characterize those rings $R$ such that $M_{R}(G)=\operatorname{End}_{R} G$, for every $R$-module $G$.

We let $\mathscr{R}$ denote the collection of rings satisfying (1) and $\mathscr{E}$ denote the collection of rings satisfying (2). Of course $\mathscr{E} \subseteq \mathscr{R}$. We show in the next section that in fact, $\mathscr{E}=\mathscr{R}$.

The problem then remains to characterize the class $\mathscr{R}$. It is the objective of this paper to initiate such an investigation. We collect information about $\mathscr{R}$ and present some classes of rings in $\mathscr{R}$. In particular we completely characterize the Artinian rings in $\mathscr{R}$. For a ring $R$ and an abelian group $G$ let $r x=0$ for all $r \in R, x \in G$. Then $M_{R}(G)=M_{0}(G)$ which is a nonring whenever

Received by the editors January 17, 1990 and, in revised form, March 12, 1990.

1980 Mathematics Subject Classification (1985 Revision). Primary 16A65, 16A76; Secondary $16 \mathrm{~A} 42$.

Portions of this work were done while the second author was a Fulbright Research Scholar at Johannes-Kepler-Universität Linz. The author wishes to express his gratitude to the Fulbright Commission for financial assistance and to Universität-Linz for their most generous hospitality. 
$|G| \geq 3$. Thus we make the following

Conventions. All rings have identity 1 , all modules are unitary, and all homomorphisms are identity preserving.

\section{General Results}

Let $R$ be a ring and $G$ an $R$-module. It is well known that $M(G)=G^{G}=$ $\{f: G \rightarrow G\}$ is a near-ring with respect to function addition and function composition. (We refer the reader to the books by Meldrum [4] and P:lz [5] for near-ring information.) The above defined near-ring $M_{R}(G)$ is a subnear-ring of $M(G)$ with the identity function as identity element. Moreover, $M(G)$ is an $R$-module under the action $(r f)(x)=r(f(x)), r \in R, f \in M(G), x \in G$. As above let $\mathscr{E}$ denote the class of all rings $R$ such that $M_{R}(G)=\operatorname{End}_{R}(G)$ for each $R$-module $G$, and let $\mathscr{R}$ denote the class of all rings $R$ such that $M_{R}(G)$ is a ring for each $R$-module $G$.

Theorem II.1. $\mathscr{E}=\mathscr{R}$.

Proof. Since $\mathscr{E} \subseteq \mathscr{R}$ it suffices to establish the converse. Let $R \in \mathscr{R}$. To each $f \in M_{R}(G)$ we associate a map $\hat{f}: M(G) \rightarrow M(G)$ where $\hat{f}(\varphi)=f \circ \varphi, \varphi \in$ $M(G)$. Since $\hat{f}(r \varphi)=f \circ r \varphi=r(f \circ \varphi)=r \hat{f}(\varphi)$ we see that $\hat{f} \in M_{R}(M(G))$. Now $M_{R}(M(G))$ is a ring since $R \in \mathscr{R}$; hence $\hat{f} \circ(\alpha+\beta)=\hat{f} \circ \alpha+\hat{f} \circ \beta$ for each $\alpha, \beta \in M_{R}(M(G))$. Therefore, for $\varphi \in M(G), \hat{f}(\alpha(\varphi)+\beta(\varphi))=\hat{f}(\alpha(\varphi))+$ $\hat{f}(\beta(\varphi))$ which in turn gives $f(\alpha(\varphi)(x)+\beta(\varphi)(x))=f(\alpha(\varphi)(x))+f(\beta(\varphi)(x))$ for each $x \in G$.

Now let $\psi \in M(G)$. The map $\bar{\psi}$ defined by $\bar{\psi}(\varphi)=\varphi \circ \psi, \varphi \in M(G)$ is in $M_{R}(M(G))$ since $\bar{\psi}(r \varphi)=r \varphi \circ \psi=r(\varphi \circ \psi)=r \bar{\psi}(\varphi)$. Given any $x_{1}, x_{2}$ in $G$, there exist $\psi_{1}, \psi_{2}$ in $M(G)$ such that $\psi_{1}(x)=x_{1}, \psi_{2}(x)=x_{2}$ for all $x \in G$. Thus for $\varphi=$ id in $M(G), \bar{\psi}_{i}(\varphi)(x)=x_{i}, i=1,2$. Hence $f\left(x_{1}+x_{2}\right)=f\left(x_{1}\right)+f\left(x_{2}\right)$ so $f \in \operatorname{End}_{R}(G)$, i.e., $M_{R}(G)=\operatorname{End}_{R}(G)$.

We next determine some properties for the class $\mathscr{R}$.

Theorem II.2. Let $S \in \mathscr{R}$ and let $\varphi: S \rightarrow R$ be a homomorphism. Then $R \in \mathscr{R}$.

Proof. Let $G$ be an $R$-module. Then as usual, $G$ is a (unitary) $S$-module via $s * g=\varphi(s) \cdot g, s \in S, g \in G$. For $f \in M_{R}(G), s \in S, g \in G$ we have $f(s * g)=f(\varphi(s) g)=\varphi(s) f(g)=s * f(g)$ so $f \in M_{S}(G)$. Since $S \in \mathscr{R}$, $M_{S}(G)$ is a ring and hence so is $M_{R}(G)$, i.e., $R \in \mathscr{R}$.

Corollary II.3. (i) Let $S \in \mathscr{R}$. If $S$ can be embedded in a ring $R$ (preserving the identity), then $R \in \mathscr{R}$.

(ii) If a subdirect product of rings $R_{\alpha}, \alpha \in A$, is in $\mathscr{R}$ then each $R_{\alpha} \in \mathscr{R}$.

(iii) If $R \in \mathscr{R}$ then $R^{X} \in \mathscr{R}$ for each set $X$.

(iv) If rad is any radical for rings then $R \in \mathscr{R}$ implies that the "semisimple part" $R / \operatorname{rad}(R)$ is in $\mathscr{R}$.

Proof. (i), (ii), and (iv) follow from II.2 while (iii) follows from (i) via the identity preserving map $r \mapsto(r, r, \ldots)$. 
Theorem II.4. Let $R$ be the group direct sum of subrings $R_{1}, R_{2}, \ldots, R_{n}$ which (as rings) are in $\mathscr{R}$. Then $R \in \mathscr{R}$.

Proof. Let $1=r_{1}+r_{2}+\cdots+r_{n}$ be the decomposition of the identity $1 \in R$ and let $1_{i}$ denote the identity of $R_{i}, i=1, \ldots, n$. If $G$ is an $R$-module then each $G_{i}:=1_{i} G$ is (unitary) $R_{i}$-module. Also, for $f \in M_{R}(G), f\left(G_{i}\right)=f\left(1_{i} G\right) \subseteq$ $1_{i} f(G) \subseteq G_{i}, i=1,2, \ldots, n$. Thus $\Phi: M_{R}(G) \rightarrow M_{R_{1}}\left(G_{1}\right) \oplus \cdots \oplus M_{R_{n}}\left(G_{n}\right)$ defined by $\Phi(f)=\left(\left.f\right|_{G_{1}}, \ldots,\left.f\right|_{G_{n}}\right)$ is a near-ring homomorphism. If $f \in \operatorname{ker} \Phi$ then $f$ is the zero map on each $G_{i}$. Therefore for $x \in G, f(x)=1 \cdot f(x)=$ $r_{1} f(x)+\cdots+r_{n} f(x)=f\left(r_{1} x\right)+\cdots+f\left(r_{n} x\right)=0$, so $\operatorname{ker} \Phi=\{0\}$ and $\Phi$ is an embedding. Since $M_{R_{i}}\left(G_{i}\right)$ is a ring, for each $i, M_{R}(G)$ is a ring, so $R \in \mathscr{R}$.

Corollary II.5. Let $R_{1}, R_{2}, \ldots, R_{n}$ be rings and let $R=R_{1} \oplus \cdots \oplus R_{n}$, the direct sum of rings. Then $R \in \mathscr{R}$ if and only if $R_{i} \in \mathscr{R}, i=1,2, \ldots, n$.

Proof. If $R \in \mathscr{R}$, each $R_{1} \in \mathscr{R}$ from II.2 while the converse follows from the previous theorem.

Let $E=\left\{e_{1}, e_{2}, \ldots, e_{n}\right\}$ be a set of mutually orthogonal idempotents of the ring $R$ with $1=\sum_{i=1}^{n} e_{i}$. We say $E$ is a complete set of orthogonal idempotents. We define a relation $\sim$ on $E$ by $e_{i} \sim e_{j}$ if $e_{i} R$ and $e_{j} R$ are isomorphic as $R$-modules $\left(e_{i} R \cong_{R} e_{j} R\right)$. It is clear that $\sim$ is an equivalence relation on $E$. We let $m(E)=\min \{|B| \mid B$ is an equivalence class with respect to $\sim\}$. The following well-known result determines when the $R$-modules $e_{i} R$ and $e_{j} R$ are isomorphic.

Lemma II.6 [1, p. 51]. Let $e_{1}, e_{2}$ be idempotents of a ring $R$. Then $e_{1} R \cong_{R} e_{2} R$ if and only if there exist $e_{12}, e_{21}$ in $R$ such that $e_{12} e_{21}=e_{1}, e_{21} e_{12}=e_{2}$, $e_{1} e_{12} e_{2}=e_{12}$, and $e_{2} e_{21} e_{1}=e_{21}$. (As pointed out in [1], the first two conditions suffice.)

Our next result gives a very useful criterion for determining many rings in $\mathscr{R}$.

Theorem II.7. Let $R$ be a ring. If $R$ has a complete set $E=\left\{e_{i j}\right\}$ of orthogonal idempotents with $m(E) \geq 2$, then $R \in \mathscr{R}$.

Proof. For $e_{i j} \in E$, let $\bar{e}_{i j}$ denote the equivalence class determined by $e_{i j} \in E$. Then without loss of generality we have

$$
R=\left(e_{11} R \oplus \cdots \oplus e_{1 j_{1}} R\right) \oplus \cdots \oplus\left(e_{k 1} R \oplus \cdots \oplus e_{k j_{k}} R\right)
$$

where $e_{i j} \in \bar{e}_{i 1}, i=1,2, \ldots, k, 1=e_{11}+\cdots+e_{k j_{k}}$, and $j_{i} \geq 2$ for all $i$. Let $G$ be an $R$-module and let $G_{i j}:=e_{i j} G$. Then $G=G_{11} \oplus \cdots \oplus G_{k j_{k}}$, a group direct sum. Let $g_{11}, \ldots, g_{k j_{k}} \in G, f \in M_{R}(G)$ and consider $g=$ $f\left(e_{11} g_{11}+\cdots+e_{k j_{k}} g_{k j_{k}}\right)-f\left(e_{11} g_{11}\right)-\cdots-f\left(e_{k j_{k}} g_{k j_{k}}\right)$. Using the orthogonality, we find for each $e_{i j} \in E, e_{i j} g=0$; hence $1 \cdot g=0$. It remains to show that $f\left(e_{i j} g_{1}+e_{i j} g_{2}\right)=f\left(e_{i j} g_{1}\right)+f\left(e_{i j} e_{2}\right)$ for all $e_{i j} \in E, g_{1}, g_{2} \in G$. Let $e_{i j}^{\prime} \in \bar{e}_{i j}$, $e_{i j} \neq e_{i j}^{\prime}$. For ease of notation we let $e_{1}=e_{i j}, e_{2}=e_{i j}^{\prime}$. From Lemma II.6, 
there exist $e_{12}, e_{21}$ in $R$ with $e_{12} e_{21}=e_{1}, e_{21} e_{12}=e_{2}, e_{1} e_{12} e_{2}=e_{12}$, and $e_{2} e_{21} e_{1}=e_{21}$. Then

$$
\begin{aligned}
f\left(e_{1} g_{1}+e_{1} g_{2}+e_{2} e_{21} g_{2}\right) & =f\left(e_{1} g_{1}+e_{12} e_{2} e_{21} g_{2}+e_{2} e_{21} g_{2}\right) \\
& =\left(1+e_{12}\right) f\left(e_{1} g_{1}+e_{2} e_{21} g_{2}\right) \\
& =\left(1+e_{12}\right)\left(f\left(e_{1} g_{1}\right)+f\left(e_{2} e_{21} g_{2}\right)\right) \\
& =f\left(e_{1} g_{1}\right)+f\left(e_{2} e_{21} g_{2}\right)+f\left(e_{12} e_{2} e_{21} g_{2}\right),
\end{aligned}
$$

so we see that $f\left(e_{1} g_{1}+e_{1} g_{2}+e_{2} e_{21} g_{2}\right)=f\left(e_{1} g_{1}\right)+f\left(e_{1} g_{2}\right)+f\left(e_{2} e_{21} g_{2}\right)$. But $f\left(e_{1} g_{1}+e_{1} g_{2}+e_{2} e_{21} g_{2}\right)=f\left(e_{1} g_{1}+e_{1} g_{2}\right)+f\left(e_{2} e_{21} g_{2}\right)$ by the first part of the proof, so the result follows.

As an application of this result we show that $\mathscr{R}$ is closed with respect to arbitrary products of matrix rings of size at least two. We remark that it is unknown to the authors if $\mathscr{R}$ is closed under arbitrary products of rings in $\mathscr{R}$.

To fix some notation we let $\mathbf{M}_{n}(S)$ denote the ring of $n \times n$ matrices over $S$. Further, let $(i, j),(k, l), i, j, k, l \in\{1,2, \ldots, n\}$ be positions located on some diagonal of the $n \times n$-board for matrices of $\mathbf{M}_{n}(S)$. Then $M((i, j),(k, l))$ will denote the matrix with 1's on this diagonal between and including $(i, j),(k, l)$, and 0's elsewhere. We abbreviate $M((i, i),(j, j))$ by $M(i, j)$ and $M(i, i)$ by $M(i)$.

Theorem II.8. Let $\left\{R_{\alpha} \mid \alpha \in A\right\}$ be a collection of rings, $\left\{n_{\alpha} \mid \alpha \in A\right\}$ a collection of integers with $n_{\alpha} \geq 2$, and let $R=\prod_{\alpha} \mathbf{M}_{n_{\alpha}}\left(R_{\alpha}\right)$. Then $R \in \mathscr{R}$. In particular, for any ring $R$, if $n \geq 2, M_{n}(R) \in \mathscr{R}$.

Proof. We define a complete set $E=\left\{e_{1}, \ldots, e_{5}\right\}$ of orthogonal idempotents as follows. If $n_{\alpha}$ is odd let $e_{k}(\alpha)=M(k)$ for $k \in\{1,2,3\}, e_{4}(\alpha)=0=e_{5}(\alpha)$ if $n_{\alpha}=3$ and $e_{4}(\alpha)=M\left(4,\left(n_{\alpha}+3\right) / 2\right)$ and $e_{5}(\alpha)=M\left(\left(n_{\alpha}+5\right) / 2, n_{\alpha}\right)$ for $n_{\alpha}>3$. If $n_{\alpha}$ is even, let $e_{k}(\alpha)=0$ for $k \in\{1,2,3\}, e_{4}(\alpha)=M\left(1, n_{\alpha} / 2\right)$, and $e_{5}(\alpha)=M\left(\left(n_{\alpha}+2\right) / 2, n_{\alpha}\right)$. One then verifies that $E$ is a complete set of orthogonal idempotents. Let $f, g \in R$ be defined by

$$
\begin{aligned}
& f(\alpha)=M\left(\left(4, \frac{n_{\alpha}+5}{2}\right),\left(\frac{n_{\alpha}+3}{2}, n_{\alpha}\right)\right), \\
& g(\alpha)=M\left(\left(\frac{n_{\alpha}+5}{2}, 4\right),\left(n_{\alpha}, \frac{n_{\alpha}+3}{2}\right)\right) \quad \text { if } n_{\alpha} \text { is odd and } n_{\alpha}>3, \\
& f(\alpha)=0=g(\alpha) \quad \text { if } n_{\alpha}=3,
\end{aligned}
$$

and

$$
\begin{aligned}
& f(\alpha)=M\left(\left(1, \frac{n_{\alpha}+2}{2}\right),\left(n_{\alpha} / 2, n_{\alpha}\right)\right), \\
& g(\alpha)=M\left(\left(\frac{n_{\alpha}+2}{2}, 1\right),\left(n_{\alpha}, n_{\alpha} / 2\right)\right) \quad \text { if } n_{\alpha} \text { is even. }
\end{aligned}
$$

From this we see that $f g=e_{4}$ and $g f=e_{5}$; hence $e_{4} R \cong{ }_{R} e_{5} R$ by the remark in II.6. Moreover, $e_{1} R \cong_{R} e_{2} R \cong_{R} e_{3} R$ so $m(E)=2$, unless $n_{\alpha}=3$ for all $\alpha \in A$ in which case $m(E)=3$. Therefore $R \in \mathscr{R}$. 
Corollary II.9. Let $R$ be a ring. Then $m(E) \geq 2$ for some complete set $E$ of orthogonal idempotents if and only if $R$ contains a subring $S$ such that $1 \in S$ and $S$ is a direct sum of ideals $I_{k}$ which (as rings) are isomorphic to full matrix rings of size at least 2.

Proof. Let $E=\left\{e_{1}, \ldots, e_{n}\right\}$ be a complete set of orthogonal idempotents such that $m(E) \geq 2$ and let $E_{1}, \ldots, E_{t}$ denote the equivalence classes with respect to $\sim$. If $I_{k}=\sum\left\{e_{i} R e_{j} \mid e_{i}, e_{j} \in E_{k}\right\}, k \in\{1,2, \ldots, t\}$, then $S=I_{1} \oplus \cdots \oplus I_{t}$ is a subring of $R$ and $1 \in S$. We note that $\sum_{e_{i} \in E_{k}} e_{i}$ is the identity for $I_{k}$. Since $I_{r} I_{s}=\{0\}$ for $r \neq s$, each $I_{k}$ is an ideal of $S$. Let $e_{k} \in E_{k}$. Since $e_{k} R \cong_{R} e_{j} R$ for each $e_{j} \in E_{k}$, there exist $e_{k j}, e_{j k}$ with the properties of Lemma II.6. We define $e_{i j}=e_{i k} e_{k j}$ and observe that $e_{i j}=e_{i} e_{i j} e_{j}$ and $e_{j i}=e_{j} e_{j i} e_{i}$; hence $e_{i j}, e_{j i} \in I_{k}$. As in [1, p. 52] $\left\{e_{i j}\right\}$ is a set of matrix units for $I_{k}$ so $I_{k}$ is a matrix ring of size at least 2 since $\left|E_{k}\right| \geq 2$. For the converse, it follows from Theorem II.8 that $m(E) \geq 2$ for some complete set $E$ of orthogonal idempotents of $S$. Since $1 \in S$ our statement follows.

We now turn to a characterization for a rather large class of rings, which includes Artinian rings, to be in $\mathscr{R}$. We need first a lemma which gives a necessary condition for a ring to be in $\mathscr{R}$.

Lemma II.10. Let $\varphi: R \rightarrow S$ be a homomorphism such that $S$ is integral, i.e., $S$ has no divisors of zero. Then $R \notin \mathscr{R}$.

Proof. From Theorem II.2, it suffices to show $S \notin \mathscr{R}$. Let $G$ denote the $S$-module $S \oplus S$ and let $X=(S \oplus\{0\}) \backslash\{(0,0)\}$. Then $s\left(s_{1}, s_{2}\right) \in X$ implies $s s_{2}=0$. Since $(0,0) \notin X, s \neq 0$ so $\left(s_{1}, s_{2}\right) \in X$. It is straightforward to verify that $X$ satisfies the conditions of Theorem II.2 of [3]. Hence $M_{S}(G)$ is not a ring, so $S \notin \mathscr{R}$.

As a corollary we obtain further necessary conditions for a ring $R$ to be in $\mathscr{R}$.

Corollary II.11. (i) If there exists a homomorphism $\psi: R \rightarrow S$ where $S$ is commutative then $R \notin \mathscr{R}$.

(ii) If $R$ has no nonzero nilpotent elements then $R \notin \mathscr{R}$.

Proof. (i) Since $S$ is commutative, $S$ has a nonzero integral homomorphic image. Since $S \notin \mathscr{R}, R \notin \mathscr{R}$.

(ii) If $R$ has no nonzero nilpotent elements then again we find that $R$ has a nonzero integral homomorphic image [6, p. 202].

We recall [6, p. 217] that a ring $R$ with Jacobson radical $J(R)$ is semiperfect if $R / J(R)$ is semisimple Artinian and $J(R)$ is idempotent lifting. In particular every Artinian ring is semiperfect. We use Theorem II.7 to completely characterize those semiperfect rings in $\mathscr{R}$.

Theorem II.12. Let $R$ be a semiperfect ring. The following are equivalent:

(i) $R \in \mathscr{R}$; 
(ii) $R / J(R) \in \mathscr{R}$;

(iii) $R / J(R)$ is the direct product of $n_{i} \times n_{i}$ matrix rings over division rings $D_{i}$ with $n_{i} \geq 2$ for each $i$.

Proof. (i) $\Rightarrow$ (ii) follows from Corollary II.3 (iv). Since idempotents in $R / J(R)$ can be lifted to $R$ and since each $n_{i} \geq 2$, there is a complete set $E$ of idempotents in $R$ with $m(E) \geq 2$. Hence $R \in \mathscr{R}$ and (iii) $\Rightarrow$ (i). Suppose now $R / J(R)$ is in $\mathscr{R}$. Since $R$ is semiperfect, $R / J(R)$ is the direct product of a finite number of $n_{i} \times n_{i}$-matrix rings over division rings $D_{i}$. Since $\bar{R}=R / J(R)$ is in $\mathscr{R}$ by hypothesis, $\bar{R}$ has no nonzero integral homomorphic images. Thus we must have $n_{i} \geq 2$ for all $i$.

\section{Miscellaneous REMARKS}

In this section we collect a few remarks about rings in $\mathscr{R}$. We start out with an example which shows that the converse of Corollary II.3 (ii) does not hold, i.e., we show that a subdirect product of rings in $\mathscr{R}$ need not be in $\mathscr{R}$.

Example III.1. Let $R:=\left\{\left(A_{1}, A_{2}, \ldots\right) \in \prod_{\mathbf{N}} \mathbf{M}_{2}(\mathbf{Z}) \mid A_{n}\right.$ is a diagonal matrix except for finitely many $n\}$. Then $R$ is a subdirect product of the rings $\mathbf{M}_{2}(\mathbf{Z})$ which are in $\mathscr{R}$. But $R \notin \mathscr{R}$ since $I:=\left\{\left(A_{1}, A_{2}, \ldots\right) \in R \mid A_{n}=0\right.$ for all but finitely many $n\}$ is an ideal in $R$ and $R / I$ is commutative.

As we have seen, no division ring is in $\mathscr{R}$. One next investigates which simple rings are in $\mathscr{R}$. If $R$ is a simple ring with a minimal left ideal then from [1, p. 88] or [6, p. 157] $R$ is a matrix ring of size at least 2 over a division ring. Thus $R \in \mathscr{R}$. However, not every simple ring which is not a division ring is in $\mathscr{R}$. For example, we let $R$ be the ring of differential polynomials over a field. Then $R$ is a simple ring with no minimal left ideals, but $R$ is integral so $R \notin \mathscr{R}$. On the other hand, $\mathscr{R}$ does contain some simple rings without minimal left ideals. In fact, let $V$ be any vector space of countable dimension over a division ring $D$ and let $I$ be the ideal of $\operatorname{End}_{D} V$ consisting of those linear transformations of $V$ of finite dimensional range. We show End $_{D} V \in \mathscr{R}$. We actually show that for any vector space $W$ over $D$ for which $\operatorname{dim}_{D} W \geq 2$, End $_{D} W \in \mathscr{R}$. If $W$ is finite dimensional then the result follows from Theorem II.8. Therefore we take $W$ to be infinite dimensional over $D$ with basis $B$. Since $B$ is infinite, there exist disjoint subsets $B_{1}, B_{2}$ of $B$ with $B=B_{1} \cup B_{2}$ and a bijection $\sigma: B_{1} \rightarrow B_{2}$. For $x \in B_{1}$, let $e_{1}(x)=x$ and for $x \in B_{2}$, let $e_{1}(x)=0$. Extend $e_{1}$ linearly to obtain an endomorphism $e_{1} \in \operatorname{End}_{D} W$. In the same manner we get $e_{2} \in \operatorname{End}_{D} W, e_{2}(x)=0, x \in B_{1}$ and $e_{2}(x)=x, x \in B_{2}$. Then $1_{W}=e_{1}+e_{2}, e_{1}$ and $e_{2}$ are idempotents and $e_{i} e_{j}=0$ for $i \neq j$.

Similarly, define $e_{12} \in$ End $_{D} W$ by $e_{12}(x)=0, x \in B_{1}, e_{12}(x)=\sigma^{-1}(x)$ for $x \in B_{2}$, and $e_{21} \in \operatorname{End}_{D} W$ by $e_{21}(x)=\sigma(x), x \in B_{1}$, and $e_{21}(x)=0$, $x \in B_{2}$. Then $e_{12} e_{21}=e_{1}$ and $e_{21} e_{12}=e_{2}$. From Theorem II.7 we see that End $_{D} W \in R$. 
We return to our special case and note that since $\operatorname{End}_{D} V \in \mathscr{R}$ so does End $_{D}(V) / I$. But this is a simple ring with no minimal left ideals.

In our final result we present an interesting characterization of $2 \times 2$ matrix rings. It is unknown to the authors if this result is new, but we have not been able to locate it in the literature.

Theorem III.2. For a ring $R$ the following are equivalent:

(i) $R$ is a ring of $2 \times 2$ matrices over some ring $S$.

(ii) There exist elements $x, y \in R$ such that $x^{2}=y^{2}=0$ and $x+y$ is invertible.

Proof. (i) $\Rightarrow$ (ii). If $\left\{e_{i j} \mid 1 \leq i, j \leq 2\right\}$ is a set of matrix units for $R$, then $e_{12}^{2}=e_{21}^{2}=0$ and $\left(e_{12}+e_{21}\right)^{2}=1$.

(ii) $\Rightarrow(\mathrm{i})$. Suppose that $(x+y) r=r(x+y)=1$. Then $x y r=x$ and $r x y=$ $y$, so $r x=y r$. Also, $r y x=x, y x r=y$, hence $r y=x r$. Consequently $x r+$ $r x=1$. But then $x r x=x$ and $(r x)^{2}=r x$. Further $r x \neq 1$ and $r x \neq 0$ since $r$ is invertible and $x \neq 0$. Therefore $r x$ is a nontrivial idempotent. Similarly $r y=x r$ is a nontrivial idempotent. Now let $e_{11}=r x, e_{22}=r y, e_{12}=r^{2} y$, and $e_{21}=x$. Then $e_{12}^{2}=r^{2} y r r y=r^{2} r x x r=0, e_{12} e_{21}=\operatorname{rryx}=r x r x=r x=e_{11}$, $e_{21} e_{12}=x r r y=(r y)^{2}=r y=e_{22}$, and $e_{11} e_{22}=r x r y=r x x r=0$. In fact, one verifies that $e_{i j} e_{k l}=\delta_{j k} e_{i l}$. Thus $\left\{e_{i j} \mid 1 \leq i, j \leq 2\right\}$ is a set of matrix units for $R$. Our statement now follows from [1, p. 52].

Thus a ring satisfying condition (ii) of the above theorem must be in $\mathscr{R}$. We also note that all of our examples of rings in $\mathscr{R}$ have nontrivial idempotents, hence the following question.

Question $A$. Are there rings in $\mathscr{R}$ with no nontrivial idempotents?

We conclude with a related question.

Question $B$. If $R \in \mathscr{R}$, is $m(E) \geq 2$ for some complete set $E$ of orthogonal idempotents in $R$ ?

\section{REFERENCES}

1. N. Jacobson, The structure of rings, Amer. Math. Soc. Colloq. Publ., vol. 37, Amer. Math. Soc., Providence, RI, 1964.

2. C. J. Maxson and K. C. Smith, Simple near-ring centralizers of finite rings, Proc. Amer. Math. Soc. 75 (1979), 8-12.

3. C. J. Maxson and A.P.J. van der Walt, Centralizer near-rings over free ring modules, J. Austral. Math. Soc. (to appear).

4. J. D. P. Meldrum, Near-rings and their links with groups, Research Notes in Math., vol. 134, Pitman Publ. Co., London, 1986.

5. G. F. Pilz, Near-rings, 2nd ed., North Holland, Amsterdam, 1983.

6. L. H. Rowen, Ring theory, Vol. I., Pure and Appl. Math., vol. 127, Academic Press, San Diego, 1988.

(P. Fuchs and G. Pilz) Institut für Mathematik, Johannes KePler Universität, A-4040 LiNZ, AUSTRIA

(C. J. Maxson) Department of Mathematics, Texas A\&M University, College Station, TEXAS 77843 